\title{
Renal Angioembolisation in the Management of Bleeding Post Percutaneous Nephrolithotomy- A Retrospective Study
}

\section{ABSTRACT}

Introduction: Renal Angioembolisation (RAE) plays a vital role in the treatment of renal bleeding after Percutaneous Nephrolithotomy (PCNL). Renal pseudoaneurysm and arteriovenous fistulas following extended haemorrhages after PCNL are the most serious complications of this operation.

Aim: To retrospectively analyse the patients for the management of bleeding post PCNL and to analyse the efficacy of renal angioembolisation.

Materials and Methods: This was a retrospective study conducted from from May 2019 to June 2020, on a total of 32 patients, who underwent PCNL for nephrolithiasis, experienced haematuria and were referred to interventional Radiology Department of tertiary care teaching hospital for renal angioembolisation. The patients who underwent a postoperative Computed Tomography (CT) scan to assess the cause of bleeding were included. Past medical records of all 32 patients were collected in terms of patient particulars, surgical details of PCNL, development of post PCNL haematuria between $5^{\text {th }}$ and $14^{\text {th }}$ postoperative days as a complication necessitating a hospital admission, digital subtraction angiographic findings, types of embolisation materials used and follow-up information. Data was analysed statistically using Sigmaplot software version 10.0 and represented in the form of percentages for all the study parameters.

Results: Of the 32 patients who were referred for renal angioembolisation in management of bleeding after PCNL, there were 21 males and 11 females with average age of $45.6 \pm 11.2$ years. Right kidney was involved in 14 cases $(43.7 \%)$ and left kidney was involved in 18 cases $(56.3 \%)$. A total of 31 patients revealed abnormal angiographic findings with Pseudoaneurysms (PA) in 29 (90.6\%), arteriovenous fistula in $2(6.3 \%)$ patients. One patient $(3.1 \%)$ showed negative findings. A total of 31 patients underwent successful embolisation and haemostasis achieved. A total of 28 patients were embolised with metallic coils, Polyvinyl Alcohol Particles (PVA) with gelatine sponge, one patient with metallic coils and liquid embolic agent and two patients with metallic coils and gelatine sponges. There was $100 \%$ success rate and the patients were followed from first to 18 months for any recurrence of bleeding.

Conclusion: Renal angioembolisation is minimally invasive, extremely safe and effective to stop renal bleeding after PCNL.

\section{INTRODUCTION}

For renal stones and some upper ureteric stones, PCNL is the treatment of choice. It has been carried out since the 1980s, with average success rates reaching 90\% [1]. Technological and instrumental advances have decreased the complication rates associated with this procedure. However, percutaneous renal surgery is identified with complications such as haemorrhage, experienced in $1-23 \%$ of cases [2].

A well-known cause of bleeding after PCNL is renal vessel damage with the subsequent development of arteriovenous fistulas or PA. Bleeding associated with PCNL is often considered to be the result of damage to the lower or middle calyceal regions of either the anterior or posterior segmental arteries [3]

Haematuria is a considerable and lethal PCNL complicating factor that requires a precise management [4,5]. Controlling severe bleeding was ineffective with conservative treatment, surgical partial nephrectomy and disease-side nephrectomy procedures. The surgical resection rate in response to bleeding is more than $50 \%$, although such procedures have the drawbacks of severe damage and complications [6]. More significantly, some patients suffer reduced renal function and renal damage is further exacerbated [7].

In the management of renal bleeding after PCNL, renal angioembolisation has played a crucial role. The location of the bleeding, the volume of bleeding and the embolisation procedure can be calculated both in a timely and precise manner to effectively stop the bleeding at the same time while optimising the safety of the renal function.
It is a marginally invasive procedure with high sensitivity and efficacy, hence reducing patient morbidity [8]. Renal angioembolisation is both a kidney-sparing and a lifesaving treatment. In different patient series, the success rates of RAE vary from $85 \%$ to $100 \%$ [9]. In order to achieve good results, the choice of embolisation material is critical and relies on the accessibility, size and flow pattern of the vessels to be occluded [10]. Ideally, the upstream and downstream vessels of the pseudoaneurysm should be concealed by embolisation. Metallic or microcoils, accompanied by gelfoam, autologous blood clot or glue, are known to be first choice agents. Embolisation complications are uncommon and include dissection of the renal artery, migration of the coil, post-embolisation syndrome, non-target embolisation, and renal function loss [11].

Embolisation triggers a 0-20\% (mean deficit, 9\%) immediate parenchymal perfusion deficit and a permanent 0-10\% perfusion deficit (mean deficit, 5\%). In most cases, however, there is a temporary spike in serum creatinine that returns within 10 days of the operation to normal pre-injury levels [12]. In patients with solitary kidneys, short-term deleterious effects are more pronounced. Recent studies on embolisation procedures have used most advanced exceptionally super selective techniques and the use of platinum microcoils can preserve functional renal parenchyma [13,14]. Simplicity of use, ease of control, and protection for patients are the advantages of Interventional Medicine in the control of bleeding, along with strong reproducibility. The current study retrospectively analyses the patients who underwent renal angioembolisation in the management of bleeding after PCNL and this may provide practical experience for further diagnosis and treatment of these conditions. 


\section{MATERIALS AND METHODS}

The current retrospective study was conducted in the Department of Radiology, Maharajah's Institute of Medical Sciences (MIMS), Nellimarla, Vizianagaram district, Andhra Pradesh, India. A total of 32 patients were referred to interventional Radiology Department, tertiary care teaching hospital for renal angioembolisation were reviewed from January 2012 to December 2018. The present study was done from May 2019 to June 2020. All patients provided written informed consent for the use of their data in clinical research.

Inclusion criteria: The data of the patients, who underwent PCNL for nephrolithiasis and experienced haematuria and underwent a postoperative CT scan, to assess the cause of bleeding were included in the study.

Exclusion criteria: The data of patients with kidney anomalies (e.g., horseshoe kidney, renal hypoplasia, polycystic kidney disease, calyceal diverticulum, etc.,), and patients who underwent simultaneous bilateral procedures and patients who were referred with post PCNL massive haematuria from somewhere else, were excluded from study.

Past medical records of all 32 patients were collected and data were organised systematically in terms of patient particulars, surgical details of PCNL, development of post PCNL haematuria between $5^{\text {th }}$ and $14^{\text {th }}$ postoperative days as a complication necessitating a hospital admission, digital subtraction angiographic findings, types of embolisation materials used and follow-up information. Patient characteristics including age, sex, stone location were recorded.

\section{Hospital Protocols}

A catheter for the ureteral occlusion balloon is implanted using a cystoscope. PCNL procedure has been conducted with fluoroscopic aid in the prone position. For tract dilatation, a balloon dilator (X-FORCE ${ }^{\circledR}$ N30 Nephrostomy Balloon Dilation Catheter, Bard Medical, GA, USA) and a 30F Amplatz sheath is used. For stone fragmentation and extraction, a stiff 24F nephroscope (Richard Wolf, Knittlingen, Germany) is used. Twenty-four hours after the nephrostomy tube has been removed, the per-urethral catheter is removed.

Transfemoral arterial catheterisation is performed under local anaesthesia. After vascular access is obtained, a 4- x or 5-French pigtail catheter is placed in the abdominal aorta. Digital Subtraction Angiography (DSA) is performed to evaluate the renal arteries, accessory renal arteries, lumbar arteries, and other collateral vessels. Then, angiography with a $4-\mathrm{x}$ or 5 -Fr Cobra catheter is performed to evaluate the renal artery and the location of the lesions. A 2.7-Fr microcatheter is used for super selective embolisation of the abnormal artery as distally as possible to minimise the loss of normal renal parenchyma. Depending upon the type of lesions, embolisation materials such as coils, gelatin sponge, PVAs of 300 to 500 microns, and liquid embolic agents can be used. Angiography is practiced upon embolisation to check the stenosis of the unusual arteries and to ascertain the percentage of parenchymal loss (infarction area). Just after the surgery, all of the patients will be constantly regulated to rule out the eventual rebreeding.

\section{STATISTICAL ANALYSIS}

Data was analysed statistically using Sigmaplot software version 10.0 and represented in the form of percentages for all the study parameters.

\section{RESULTS}

In the present study of 32 patients, there were 21 males and 11 females with mean age of $45.6 \pm 11.2$ years. Clinical characteristics of all patients are presented in [Table/Fig-1]. Right kidney was involved in 14 cases (43.7\%) and left kidney was involved in 18 cases (56.3\%). Angiographic findings were seen between $5^{\text {th }}$ and $14^{\text {th }}$ day of post PCNL. Persistent and recurrent haematuria was the most common presenting feature in all the 32 patients following PCNL. Thirty one patients revealed abnormal angiographic findings with PA in 29 (90.6\%), arteriovenous fistula in
2 (6.3\%) patients. One patient (3.1\%) showed negative findings. This patient was admitted with haematuria after PCNL like other patients and was referred for renal angioembolisation but his renal angiographic findings did not reveal any abnormality, hence angiographically negative findings. Renal angioembolisation was not done for the patient and he was treated conservatively with blood transfusion, there was no recurrence of haematuria on follow-up of this patient. Thirty-one patients underwent successful embolisation and haemostasis achieved. Twenty eight patients were embolised with metallic coils, PVA with gelatine sponge, one patient with metallic coils and liquid embolic agent and two patients with metallic coils and gelatine sponges. The success rate of treatment was $100 \%$. These patients were followed for 1 to 18 months for any recurrence of bleeding. There were no significant procedure related complications. [Table/Fig-2] showed the embolisation of post PCNL arteriovenous fistula in the lower pole of right kidney and [Table/Fig-3] showed the embolisation of pseudoaneurysm in the lower pole of left kidney, super selective microcatheter and coiled mass with complete exclusion of pseudoaneurysm from the circulation.

\begin{tabular}{|l|c|}
\hline Clinical category & Statistics (\%) \\
\hline Average age & $45.6 \pm 11.2$ years \\
\hline Sex (M/F) & $21 / 11(65.6 / 34.4)$ \\
\hline Location (right/left) & $14 / 18(43.7 / 56.3)$ \\
\hline Haematuria & $32(100)$ \\
\hline Abnormal findings & $29(90.6)$ \\
\hline Pseudoaneurysms & $2(6.3)$ \\
\hline Arteriovenous fistula & $1(3.1)$ \\
\hline Negative findings & $28(87.5)$ \\
\hline Embolic material used in 31 patients & $1(3.1)$ \\
\hline Metallic coils with PVA particles, gelatine sponges & $2(6.3)$ \\
\hline Metallic coils with liquid embolic agent & \\
\hline Metallic coils with gelatine sponges & \\
\hline [Table/Fig-1]: Clinical characteristics of all patients. \\
M: Male; F: Female; N=32; one patient did not undergo renal angioembolisation for haematuria \\
post PCNL due to negative renal angiographic findings
\end{tabular}
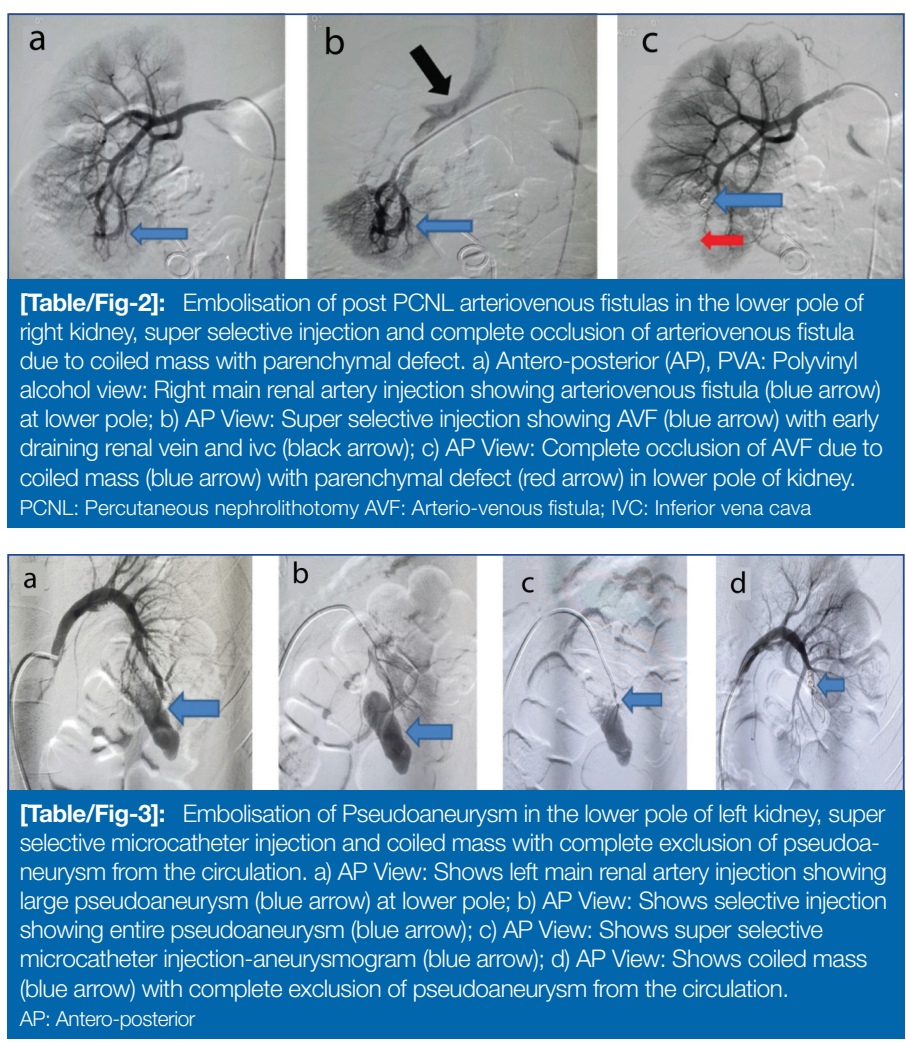

\section{DISCUSSION}

In the current retrospective study, 32 patients who developed bleeding after PCNL were treated with renal angioembolism. The success rate of treatment was $100 \%$. There were no significant procedure related 
complications. These patients were followed for 1 to 18 months for any recurrence of bleeding. Most common angiographic abnormality was renal psuedoaneurysm which accounted for 29 cases. Each renal artery has an independent blood supply area. There is almost no anastomosis between the arteries in each segment, providing an anatomical basis for super selective arterial embolisation for renal haemorrhaging [15]. Immediate haemostasis, without relapse, can be reached. In order to avoid the relapse and promote renal function efficiency, the embolisation area must therefore be located well apart from either the renal artery and near to the bleeding site [16].

There are many facets to the choosing of embolisation methods. A gelatin sponge, steel band, pva particles, and N-Butyl Cyanoacrylate (NBCA) glue are involved in arterial embolisation products. Gelatin sponges and spring coils [17] are the most frequently used materials. A medium-effective embolisation material [18] is a gelatin sponge that can quickly absorb blood. Blood can be embolised by sponge gelatin. The spring coil is a stable material for embolisation. According to the diameter of the blood vessels, different sizes of spring coils can be selected [19].

The embolisation site should be as close as possible to the bleeding site and the use of permanent embolisation material is must to achieve haemostasis and to reduce post-treatment recurrences [20]. When the vascular injury is associated with a pseudoaneurysm or an arteriovenous fistula, a metallic coil embolisation or liquid embolic agent or metallic coil with gelatine sponges is effective in completely occluding the bleeder vessel. In summary, after PCNL, renal angiography may identify the location of renal haemorrhaging. The benefits are that it is safe, minimally invasive and effective. In addition, it results in a positive prognosis and less issue, as well as a fast recovery with optimum security [21].

In this group, 28 cases were treated with metallic coils, PVA particles and gelatine sponges, two cases with metallic coils and liquid embolic agent and one case with metallic coils and gelatin sponge. No recurrence was noted in any of the cases on follow-up. A case with negative angiographic findings didn't undergo embolisation and on follow-up, the bleeding stopped spontaneously with no recurrence. There were no significant procedure related complications. Similar studies by Wen $\mathrm{S}$ et al., had reported 20 patients were embolised using metallic coils plus gelatin sponges, accounting for $47.62 \%$ [22]. Manjeet $A$ and Pawar $P$, had reported arteriovenous fistula in two patients and pseudoaneurysm in seven patients were revealed by angiography [23]. Recently, Kim HY et al., (2020) reported after PCNL, 21 patients underwent angioembolisation. Erroneous puncture was noticed in 14 (66.7\%) of PCNL angioembolised patients or even in 11 (34.4\%) of non-PCNL angioembolised patients [24].

\section{Limitation(s)}

Smaller sample size and a retrospective design may be identified as limitations of the present study.

\section{CONCLUSION(S)}

Renal angioembolisation can determine the exact site of bleed after PCNL and occlude the bleeder at the same time. It's extremely safe, minimally invasive, effective in managing bleeding after PCNL and can help preserve renal function, reduce the potential risk of nephrectomy.

\section{REFERENCES}

[1] Qilai LJ, Guo Z, Xu Y, Yang HW, Yanjun Z, Yongkang Z, et al. Experience of mini-percutaneous nephrolithotomy in the treatment of large impacted proximal ureteral stones. Urol Int. 2013;90(4):384-88.

[2] Eric T, Joe M, Thomas C, Stoller ML. Complications associated with percutaneous nephrolithotomy. Transl Androl Urol. 2012;1(4):223-28.

[3] Singh R, Kankalia SP, Sabale V, Satav V, Mane D, Mulay A, et al. Comparative evaluation of upper versus lower calyceal approach in percutaneous nephrolithotomy for managing complex renal calculi. Urol Ann. 2015;7(1):31-35.

[4] Sommer CM, Stampfl U, Bellemann N, Ramsauer S, Loenard BM, Haferkamp, et al. Patients with life-threatening arterial renal hemorrhage: CT angiography and catheter angiography with subsequent super selective embolisation. Cardio Vascular and Interventional Radiology. 2010;33(3):498-508.

[5] Canstasdemir M, Adaletli I, Cebi D, Kantarci F, Selcuk ND, Numan F. Emergency endovascular embolisation of traumatic intrarenal arterial pseudoaneurysms with N-Butyl cyanoacrylate. Clinical Radiology. 2003;58(7):560-65.

[6] Richstone L, Reggio E, Ost MC. First Prize (tie): Hemorrhage following percutaneous renal surgery: Characterization of angiographic findings. Journal of Endourology. 2008;22(6):1129-35.

[7] Saad WG, Mmdouh A. Pediatric PCNL puncture complication: Risk factors. Eur Urol. 2018;17:e805.

[8] Krishna Reddy SV, Ahammad Basha S. Outcome and complications of percutaneous nephrolithotomy as primary versus secondary procedure for renal calculi. Int Braz J Urol. 2016;42(2):262-69.

[9] Michael Schwartz J, Eric Smith B, David Trost W, Darracott Vaughan E. Renal artery embolisation: Clinical indications and experience from over 100 cases. BJU Int. 2007;99(4):881-86.

[10] Sutherland DE, Williams SB, Rice D, Jarrett TW, Engel JD. Vascular pseudoaneurysms in urology: Clinical characteristics and management. J Endourol. 2010;24(6):915-21.

[11] Cantasdemir M, Adaletli I, Cebi D, Kantarci F, Selcuk ND, Numan F. Emergency endovascular embolisation of traumatic intrarenal arterial pseudoaneurysms with n-butyl cyanoacrylate. Clin Radiol. 2003;58(7):560-65.

[12] Kidney Disease: Improving Global Outcomes (KDIGO) Acute Kidney Injury Work Group. KDIGO Clinical Practice Guideline for Acute Kidney Injury. Kidney Inter Suppl. 2012;2(Supp 1):S01-138.

[13] Inci K, Cil B, Yazici S. Renal artery pseudoaneurysm: Complication of minimally invasive kidney surgery. J Endourol. 2010;24(1):149-54.

[14] Li L, Zhang Y, Chen Y. A multicentre retrospective study of transcatheter angiographic embolisation in the treatment of delayed haemorrhage after percutaneous nephrolithotomy. Eur Radiol. 2015;25(4):1140-47.

[15] Vignali C, LonziS, Bargellini I. Vascular injuries after percutaneous renal procedures: Treatment by transcatheter embolisation. Eur Radiol. 2004;14(4):723-29.

[16] Wang HL, Xu CY, Wang HH, Xu W. Emergency transcatheter arterial embolisation for acute renal hemorrhage. Medicine (Baltimore). 2015;94(42):e1667.

[17] lerardi AM, Duka E, Lucchina N, Floridi C, De Martino A, Donat D, et al. The role of interventional radiology in abdominopelvic trauma. $\mathrm{Br} J$ Radiol. 2016;89(1061):20150866.

[18] Madhusudhan KS, Venkatesh HA, Gamanagatti S, Garg P, Srivastava DN. Interventional radiology in the management of visceral artery pseudoaneurysms: A review of techniques and embolic materials. Korean J Radiol. 2016;17(3):351-63.

[19] Katsumori T, Arima H, Asai S, Hayashi N, Miura H. Comparison of pain within $24 \mathrm{~h}$ after uterine artery embolisation with Tris-Acryl gelatin microspheres versus gelatin sponge particles for leiomyoma. Cardiovasc Intervent Radiol. 2017:40(11):1687-93.

[20] Ortiz J M, Bhattacharyya N. Management pitfalls in the use of embolisation for the treatment of severe epistaxis. Ear Nose Throat J. 2002;81(3):178-83.

[21] Zhang $X$, Zhao W, Huang J, Jiang Y, Shi Y. Clinical application value of interventional embolisation in treating renal pseudoaneurysms. J Interv Radiol. 2015;379-82.

[22] Wen S, Peng Z, Xiong Z, Guobing Z, Bensheng Z, Mingquan W, et al. Renal angiography and artery embolisation for treatment of bleeding after PCNL: Analysis of 44 cases. Int J Clin Exp Med. 2019;12(11):12963-67.

[23] Manjeet A, Pawar P. Predictors for severe hemorrhage requiring angioembolisation post percutaneous nephrolithotomy: A single-center experience over 3 years. Urol Ann. 2019;11(2):180-86.

[24] Kim HY, Kyu WL, Dong SL. Critical causes in severe bleeding requiring 'angioembolisation after percutaneous nephrolithotomy. BMC Urol. 2020;20:22. https://doi.org/10.1186/s12894-020-00594-6.

PARTICULARS OF CONTRIBUTORS:

1. Associate Professor, Department of Radiology, Maharajah's Institute of Medical Sciences (MIMS), Vizianagaram, Andhra Pradesh, India

2. Associate Professor, Department of Radiodiagnosis, Gitam Institute of Medical Sciences and Researh, Gitam Deemed to be University, Visakhapatnam, Andhra Pradesh, India.

NAME, ADDRESS, E-MAIL ID OF THE CORRESPONDING AUTHOR:

Devara Anil Kashi Vishnuvardhan,

Associate Professor, Department of Radiology, Maharajah's Institute of Medical Sciences

(MIMS), Nellimarla, Vizianagaram District, Andhra Pradesh, India.

E-mail: rajaniagis@gmail.com
PLAGIARISM CHECKING METHODS: [Jain Het al.J

- Plagiarism X-checker: Sep 24, 2020

ETYMOLOGY: Author Origin

- Manual Googling: Mar 02, 2021

- iThenticate Software: Apr 02, 2021 (10\%)

\section{AUTHOR DECLARATION:}

- Financial or Other Competing Interests: None

- Was Ethics Committee Approval obtained for this study? NA

- Was informed consent obtained from the subjects involved in the study? Yes

- For any images presented appropriate consent has been obtained from the subjects. NA

Date of Submission: Sep 23, 2020 Date of Peer Review: Jan 06, 2021 Date of Acceptance: Mar 06, 2021 Date of Publishing: Jul 01, 2021 